\title{
Vascular Access Care at Hemodialysis Unit; nurses' compliance to Infection prevention and Control Practices العناية بالوصلة الدموية بوحدة الاستصفاء الدموي ؛التزام الممرضات بممارسات الوقاية والتحكم فى العدوى الأوي
}

\author{
Dr/ Abeer Mohamed Elshatby Moursy ${ }^{1}, \mathrm{Dr} /$ Amany Youssef Sharaf ${ }^{2}$ \\ ${ }^{I}$ Assistant prof; Medical surgical Nursing department- Faculty of Nursing, Alexandria University-Egypt \\ ${ }^{2}$ Lecturer, Medical surgical Nursing department- Faculty of Nursing, Alexandria University-Egypt
}

\begin{abstract}
Patients on maintenance Hemodialysis are at high risk of acquiring vascular access infection because of their immune suppression state. So, compliance to infection prevention and control practices (IPCP) has to be investigated.

Objective: to assess hemodialysis nurses compliance to infection prevention and control practices during vascular access care.
\end{abstract}

Setting: This study was conducted at Hemodialysis units of El-Moassat, and Medical Research Institute Hospitals.

Subjects: The study subjects included all nurses responsible for Hemodialysis procedures at the above mentioned settings. Their numbers was 48 nurses (26 from El-Moassat and 22 from Medical Research Institute hospitals).

Tools: Two tools were used for data collection: The Haemodalysis Nurses Structured Questionnaire Sheet and An observational checklist.

Results: The overall percent score of nurses' knowledge about infection prevention and control practices was (61.57\%). The overall nurses' compliance to IPCP at both hospitals was poor.

Conclusion: The majority of nurses were not following principles of infection prevention and control practices during Hemodialysis vascular access care.

Recommendations: Policies and infection control procedures at HD units should be regularly checked to ensure better compliance to infection prevention and control practices.

Keywords: Haemodialysis, vascular access care, infection control practices.

\section{Introduction}

Hemodialysis (HD) is the therapy used most often among patients with end-stage renal disease ESRD (1). In Egypt, the annual incidence of ESRD is about 74 per million, and the prevalence of patients on dialysis is 264 per million population ${ }^{(2)}$. While HD therapy has improved many patients' care, arguments remain, regarding the quality of care provided to patients by dialysis facilities ${ }^{(3)}$.

In 2015, Egypt Health Issues Survey showed that 10\% of Egyptians under HD between $15-59$ years of age had been infected with HCV infection, while $7 \%$ of them had chronic hepatitis $\mathrm{C}^{(4)}$. Blood is the main source of blood borne pathogens in dialysis units. In a report issued by Young et al (2005), vascular access infection is the source of $50-73 \%$ of bacteraemia in HD patients ${ }^{(5)}$. Also, in USA, $37.5 \%$ of hospitalizations of patients on HD in 2007, were due to infection, which ranks as a principal cause of morbidity and the second leading cause of mortality ${ }^{(6)}$. Young et al (2005) postulated that vascular access complications due to infection include; endocarditis, meningitis, osteoarthritis or formation of septic emboli, and up to $36 \%$ of End Stage Kidney Disease (ESKD) deaths ${ }^{(5)}$.

Chronic HD patients are at high risk of developing vascular access infection due to the immune suppression state caused by uraemia, and the increased susceptibility to nosocomial infections due to prolonged hospitalization and surgery ${ }^{(7)}$. Also, the dialysis staffs are at high risk of developing blood borne infections due to the unsafe use of injection equipment, other medical devices, and blood products in the dialysis unit ${ }^{(8)}$.

So, several studies recommended that competent infection control practices should be established at dialysis units to prevent transmission of bacteria and viruses ${ }^{(9,10,11,12)}$. Storr et al (2005) added that competence combines nurses' knowledge about techniques that ensure prevention of cross infection, and how to practice them ${ }^{(13)}$.

In the developing countries, economical problems lead to inability of dialysis units to reach the proposed targets. In addition, infection control is not compulsory, as the available resources are usually needed for other health issues. The lack of knowledge and the way that the problem is perceived are the main factors leading to poor infection control in these countries ${ }^{(11)}$. 
Vascular Access Care at Hemodialysis Unit; nurses' compliance to Infection prevention and ..

HD units are different from other hospital wards, as HD patients have prolonged blood exposures, and usually with one nurse who cares for more than one patient at the same time, which increase the risk of infection transmission. Therefore, infection control practices as; hand hygiene, personal protective equipments (PPE), and vascular access care should be strictly recommended ${ }^{(8)}$. Those practices however are frequently questioned and have to be investigated.

The increasing prevalence of vascular access infection at HD units increase patients, and staff's risk of developing blood borne pathogens ${ }^{(7,8)}$. HD nurses have an important role in understanding, and complying to the principles of infection control. With carefully prospective studies in this area, we can infer a link between nurse's knowledge, and practice regarding infection prevention of HD vascular access. This study was designed to evaluate hemodialysis nurses' knowledge and practice of infection prevention and control during vascular access care.

Aim of the study:

To assess HD nurses' compliance to infection prevention and control practices during vascular access care.

Research question:

To what extent do HD nurses follow infection prevention and control practices during vascular access care?

\section{Materials}

Design:

A descriptive design was utilized.

Setting:

The study was conducted at the Hemodialysis units of El-Moassat and the Medical Research Institute, Alexandria University, Hospitals.

\section{Subjects:}

The sample of this study included all nurses responsible for HD procedures in the above mentioned settings. Their numbers was 48 nurses (22 from Institute of Medical Research and 26 from El-Moassat Alexandria University Hospital)

Tools:

Two tools were used for the purpose of the study.

Tool I: HD Nurses Structured Questionnaire Sheet:

An Arabic structured questionnaire sheet was developed by the researchers based on a review the relevant literature to assess HD nurses knowledge about blood borne infection, infection prevention and control practices and HD vascular access care ${ }^{(6,7,8,9,10)}$ It comprised two parts:

Part I: This part included socio-demographic data about nurses' age, marital status, level of education, years of experience, attendance, date of pre-service and in-service training programs and the persons who taught the nurses.

Part II: This part included questions about nurses' knowledge related to blood borne infection, infection prevention and control practices and vascular access care. It comprised three main subcategories regarding the following areas of knowledge:

A- Blood -borne pathogens. This subcategory included six open ended questions about types of blood -borne diseases, types of microorganisms causing AIDS and hepatitis, personnel at risk of blood borne infection at HD units, vehicles and routes of transmission among HD patients and nurses. Nurses were required to respond to items under each of the previously mentioned six questions, two score were given for (correct / complete answer), one score for (incomplete answer) and zero for (don't know). The total score of nurses' knowledge about blood- borne pathogens ranged was 12 .

B- Infection prevention and control practices at HD units. It includes 19 questions related to hand washing , gloving, gowning, wearing mask, using eye wear, needles handling and disposal, and periodical blood screening for patients and nurses. Nurses were asked to indicate the degree of importance and correct answer of each item under each of the nineteen questions on a three likert type scale; ranging from important/correct answer (2), less important/incomplete answer (1) to not important / don't know (zero). The total score value of nurses' knowledge about infection prevention and control practices was 38 .

C- HD vascular access care. This subcategory included seven open ended questions related to vascular access definition, types, practices before needle insertion, checking patency, duration for maturation, instructions given for vascular access maintenance. Nurses were required to respond to items under each of the previously mentioned seven questions. Nurses responses were graded and scored as follows; (correct and complete response) two scores, (incomplete responses) one score and (don't know) zero score. The total score of nurses' knowledge about HD vascular access care was 14. 
Vascular Access Care at Hemodialysis Unit; nurses' compliance to Infection prevention and ..

Total nurses knowledge score were estimated, then converted to mean percent score and classified as follows: Good: $>75 \%$, Fair:50-75\% and Poor: $<50 \%$.

Tool II: The HD Patients' vascular access care: infection prevention and control practices observational checklist. This observational checklist was developed by the researchers to assess nurses' practice of infection control practices during HD vascular access cannulation and decannulation. ${ }^{(6,7,8,9,10)}$. It comprised two parts;

The first part consisted of 15 items of IPCP during cannulation of arteriovenous fistula or graft .The procedure steps included hand washing, patient preparation, assessing vascular access function, signs and symptoms of infection, PPE, sterile gloving, disinfection access site, aseptic cannulation and bloodlines. Scores of 2, 1, and zero were allotted to steps (correctly and completely done), (incorrectly and incompletely done) and (not done) respectively. The total observation checklist scores were 45.

The second part consisted of 11 items of IPCP during decannulation of arteriovenous fistula or graft. The procedure steps included hand washing, wearing PPE, Wearing sterile gloves, disconnecting bloodlines and needles aseptically, disinfecting vascular site, needle handling and disposal. Scores of 2, 1, and zero were allotted to steps (correctly and completely done), (incorrectly and incompletely done) and (not done) respectively. The total observation checklist scores were out of 22.

Total nurses practice score were calculated then converted to mean percent score and classified as: Good nurses practice: $>75 \%$, Fair: $50-75 \%$ and Poor: $<50 \%$.

\section{Method}

1. An official written permission to carry out the study was obtained from the head of HD unit and the head nurse of the hospital after submitting an official letter from the Dean of the Faculty of Nursing at Alexandria.

2. Tools of the study were developed after a review of relevant literature. Then, the tools were tested for content validity by a jury of five experts in the field of medical surgical nursing. Tools reliability were tested by Cronbach's co-efficiency alpha test for tool I ( $r=0.77)$ and for tool II $(r=0.78)$.

3. A Pilot study was carried on $10 \%$ of the studied nurses after the tools were developed and before starting the data collection to test the clarity, and applicability of the developed tools.

4. Data collected throughout a period of 4 months from February to end of May 2016.

5. Every nurse was observed throughout HD procedures from initiation to termination of arteriovenous fistula or graft cannulation, using concealed continuing observation. Observation sheet items were checked for every nurse at the morning and afternoon shifts.

6. After completing all observations, nurses were asked to answer the questionnaire sheet. They were interviewed individually at nurses' office stations during break hours. The questionnaires were completed within 20 to 25 minutes each.

\section{Ethical considerations:}

Written consents were obtained from nurses before participation in the study after explaining that the collected data would be used only for study purposes, confidentiality and privacy were assured.

\section{Statistical analysis of the data}

Data were computed, fed, and analyzed using IBM SPSS software package version 20.0.Qualitative data were described using number and percent. Quantitative data were described using mean, standard deviation. Significance of the obtained results was judged at the 5\% level. The used tests were 1 - Chi-square test: For categorical variables, to compare between different groups.2 - Fisher's Exact or Monte Carlo correction: Correction for chi-square when more than $20 \%$ of the cells have expected count .3 - Paired t-test: For normally quantitative variables, to compare between two periods. 4 - Wilcoxon signed ranks test: For abnormally quantitative variables, to compare between two periods.

\section{Results}

Table (1): Distribution of the studied nurses according to their socio-demographic characteristics $(\mathrm{n}=48)$

\begin{tabular}{|c|c|c|}
\hline Socio-demographic characteristics & No & $\%$ \\
\hline $\begin{array}{c}\text { Age (years) } \\
20>30 \\
30>40 \\
40>50 \\
50-60\end{array}$ & $\begin{array}{c}9 \\
26 \\
9 \\
4\end{array}$ & $\begin{array}{c}18.8 \\
54.2 \\
18.8 \\
8.3\end{array}$ \\
\hline $\begin{array}{c}\text { Social status } \\
\text { Single } \\
\text { Married } \\
\text { Widow }\end{array}$ & $\begin{array}{c}5 \\
41 \\
2\end{array}$ & $\begin{array}{c}10.4 \\
85.4 \\
4.2\end{array}$ \\
\hline
\end{tabular}


Vascular Access Care at Hemodialysis Unit; nurses' compliance to Infection prevention and ..

\begin{tabular}{|c|c|c|}
\hline $\begin{array}{l}\text { Education level } \\
\text { Diploma } \\
\text { Bachelor } \\
\end{array}$ & $\begin{array}{l}32 \\
16 \\
\end{array}$ & $\begin{array}{l}66.7 \\
33.3 \\
\end{array}$ \\
\hline $\begin{array}{l}\text { Years of experience at HD unit (years) } \\
\quad<10 \\
10<20 \\
20<30 \\
30<40\end{array}$ & $\begin{array}{c}8 \\
25 \\
13 \\
2\end{array}$ & $\begin{array}{c}16.7 \\
52.1 \\
27.1 \\
4.2\end{array}$ \\
\hline $\begin{array}{l}\text { Attendance of HD training programs } \\
\text { Yes } \\
\text { No }\end{array}$ & $\begin{array}{c}46 \\
2 \\
\end{array}$ & $\begin{array}{c}95.8 \\
4.2\end{array}$ \\
\hline $\begin{array}{l}\text { Attendance of IPCP training programs } \\
\text { Yes } \\
\text { No }\end{array}$ & $\begin{array}{c}45 \\
3 \\
\end{array}$ & $\begin{array}{c}93.8 \\
6.3 \\
\end{array}$ \\
\hline $\begin{array}{l}\text { If yes, when }(\mathbf{n}=45) \\
\text { Pre-service } \\
\text { In-service }\end{array}$ & $\begin{array}{c}4 \\
41 \\
\end{array}$ & $\begin{array}{c}8.9 \\
91.1 \\
\end{array}$ \\
\hline $\begin{array}{l}\text { Trainers } \\
\text { Doctors } \\
\text { Head nurse } \\
\text { Colleague } \\
\text { Others } \\
\end{array}$ & $\begin{array}{c}1 \\
20 \\
23 \\
4 \\
\end{array}$ & $\begin{array}{c}2.1 \\
41.7 \\
47.9 \\
8.3 \\
\end{array}$ \\
\hline
\end{tabular}

Table (1) Illustrates that more than half of the studied nurses $(54.2 \%)$ were $30>40$ years, while only $8.3 \%$ were 50-60 years old. Also, the majority of nurses $(85.4 \%)$ were married and only one third $(33.3 \%)$ were bachelor graduates. As evident, $52.1 \%$ of nurses had $10<20$ years of experience, and the majority of them attended both HD and IPCP training programs during work.

Tables (2): Distribution of the studied nurses according to their total knowledge mean percent score about blood borne diseases, vascular access care and infection control precaution during vascular access care.

\begin{tabular}{|c|c|c|c|c|c|c|c|c|}
\hline \multirow[t]{2}{*}{ Areas of nurses knowledge } & \multicolumn{2}{|c|}{ Poor } & \multicolumn{2}{|c|}{ Fair } & \multicolumn{2}{|c|}{ Good } & \multirow[b]{2}{*}{ Mean \pm SD } & \multirow{2}{*}{$\begin{array}{c}\begin{array}{c}\text { Total Mean } \\
\% \text { score }\end{array} \\
\text { Mean } \pm \text { SD } \\
\end{array}$} \\
\hline & No. & $\%$ & No. & $\%$ & No. & $\%$ & & \\
\hline Blood-borne diseases & 14 & 29.2 & 16 & 33.3 & 18 & 37.5 & $6.81 \pm 2.66$ & $56.77 \pm 22.13$ \\
\hline b) HD vascular access and its care & 22 & 45.8 & 19 & 39.6 & 7 & 14.6 & $7.10 \pm 3.07$ & $50.74 \pm 21.91$ \\
\hline $\begin{array}{l}\text { Infection prevention and control practices } \\
\text { during vascular access care. }\end{array}$ & 8 & 16.7 & 31 & 64.6 & 9 & 18.8 & $23.40 \pm 5.30$ & $61.57 \pm 13.95$ \\
\hline - $\quad$ Hand washing & 1 & 2.1 & 29 & 60.4 & 18 & 37.5 & $5.31 \pm 0.75$ & $66.41 \pm 9.35$ \\
\hline - $\quad$ PPE & 9 & 18.8 & 26 & 54.2 & 13 & 27.1 & $18.08 \pm 5.13$ & $60.28 \pm 17.11$ \\
\hline Gloving & 4 & 8.3 & 10 & 20.8 & 34 & 70.8 & $4.54 \pm 1.20$ & $75.69 \pm 20.03$ \\
\hline Gown & 5 & 10.4 & 18 & 37.5 & 25 & 52.1 & $4.19 \pm 1.42$ & $69.79 \pm 23.73$ \\
\hline Face shield & 21 & 43.8 & 16 & 33.3 & 11 & 22.9 & $3.15 \pm 1.75$ & $52.43 \pm 29.17$ \\
\hline Goggles & 22 & 45.8 & 13 & 27.1 & 13 & 27.1 & $2.75 \pm 2.08$ & $45.83 \pm 34.64$ \\
\hline - Sharp instruments & 22 & 45.8 & 7 & 14.6 & 19 & 39.6 & $2.0 \pm 1.03$ & $50.0 \pm 25.79$ \\
\hline Periodic Blood screening for staff & 13 & 27.1 & 0 & 0.0 & 35 & 72.9 & $1.46 \pm 0.90$ & $72.92 \pm 44.91$ \\
\hline Overall knowledge & 10 & 20.8 & 30 & 62.5 & 8 & 16.7 & $\mathbf{3 7 . 3 1} \pm 8.37$ & $58.30 \pm 13.07$ \\
\hline
\end{tabular}

Score system: poor $<50 \%$ fair $50 \%-<75 \% \quad$ good $\geq 75 \%$

Table (2) the results revealed that $37.5 \%$ of the studied nurses had good knowledge about blood borne diseases and around half of the respondents (45.8\%) had poor knowledge about HD vascular access care. Also, these results revealed that $64 \%$ of nurses had fair knowledge related to infection prevention and control practices during HD vascular access care with mean \pm SD $(61.57 \pm 13.95)$. The highest percentage of the studied nurses had fair knowledge about hand washing and wearing PPE with mean percent score of $(66.41 \%$ and $60.28 \%)$ respectively. The overall Nurses' knowledge was fair with mean percent score of $(58.30 \pm 13.07)$.

Table (3): Distribution of the studied nurses' practices of infection prevention and control during cannulation of AV fistula/graft throughout morning and afternoon shifts

\begin{tabular}{|c|c|c|c|c|c|c|c|c|c|c|c|c|c|}
\hline \multirow{3}{*}{$\begin{array}{l}\text { Nurses' practices of IPC during } \\
\text { cannulation of AV fistula/graft }\end{array}$} & \multicolumn{6}{|c|}{ Morning shift } & \multicolumn{6}{|c|}{ Afternoon shift } & \multirow{3}{*}{$\mathbf{p}$} \\
\hline & \multicolumn{2}{|c|}{ Not done } & \multicolumn{2}{|c|}{$\begin{array}{c}\text { Done } \\
\text { incorrectly }\end{array}$} & \multicolumn{2}{|c|}{$\begin{array}{c}\text { Done } \\
\text { correctly }\end{array}$} & \multicolumn{2}{|c|}{ Not done } & \multicolumn{2}{|c|}{$\begin{array}{c}\text { Done } \\
\text { incorrectly }\end{array}$} & \multicolumn{2}{|c|}{$\begin{array}{c}\text { Done } \\
\text { correctly }\end{array}$} & \\
\hline & No. & $\%$ & No. & $\%$ & No. & $\%$ & No. & $\%$ & No. & $\%$ & No. & $\%$ & \\
\hline Perform Hand hygiene & 20 & 41.7 & 26 & 54.2 & 2 & 4.2 & 35 & 72.9 & 11 & 22.9 & 2 & 4.2 & $<0.001^{*}$ \\
\hline $\begin{array}{l}\text { Place access limb on a sterile drape or } \\
\text { barrier }\end{array}$ & 19 & 39.6 & 23 & 47.9 & 6 & 12.5 & 27 & 56.3 & 15 & 31.3 & 6 & 12.5 & $0.005^{*}$ \\
\hline Assess vascular access function & 22 & 45.8 & 2 & 4.2 & 24 & 50.0 & 22 & 45.8 & 3 & 6.3 & 23 & 47.9 & 0.317 \\
\hline
\end{tabular}


Vascular Access Care at Hemodialysis Unit; nurses' compliance to Infection prevention and ..

\begin{tabular}{|c|c|c|c|c|c|c|c|c|c|c|c|c|c|}
\hline $\begin{array}{l}\text { Assess S\&S of vascular access infection } \\
\text { before cannulation }\end{array}$ & 15 & 31.3 & 4 & 8.3 & 29 & 60.4 & 14 & 29.2 & 4 & 8.3 & 30 & 62.5 & 0.317 \\
\hline $\begin{array}{l}\text { Wear proper PPE (wearing overshoes, } \\
\text { wearing mask and goggles, and sterile } \\
\text { gowns) }\end{array}$ & 48 & 100.0 & 0 & 0.0 & 0 & 0.0 & 48 & 100.0 & 0 & 0.0 & 0 & 0.0 & 1.000 \\
\hline Put on new sterile gloves & 48 & 100.0 & 0 & 0.0 & 0 & 0.0 & 48 & 100.0 & 0 & 0.0 & 0 & 0.0 & 1.000 \\
\hline $\begin{array}{l}\text { Clean access arm for a minute with soap } \\
\text { and water or antiseptic soap }\end{array}$ & 33 & 68.8 & 10 & 20.8 & 5 & 10.4 & 33 & 68.8 & 10 & 20.8 & 5 & 10.4 & 1.000 \\
\hline $\begin{array}{l}\text { Cleanse access site with antiseptic } \\
\text { solution }\end{array}$ & 2 & 4.2 & 33 & 68.8 & 13 & 27.1 & 2 & 4.2 & 33 & 68.8 & 13 & 27.1 & 1.000 \\
\hline Allow skin antiseptic to dry & 13 & 27.1 & 1 & 2.1 & 34 & 70.8 & 12 & 25.0 & 1 & 2.1 & 35 & 72.9 & 0.317 \\
\hline \begin{tabular}{|l|} 
Avoid contact with fistula/ graft site \\
\end{tabular} & 17 & 35.4 & 25 & 52.1 & 6 & 12.5 & 17 & 35.4 & 25 & 52.1 & 6 & 12.5 & 1.000 \\
\hline perform cannulation aseptically & 9 & 18.8 & 30 & 62.5 & 9 & 18.8 & 8 & 16.7 & 30 & 62.5 & 10 & 20.8 & 0.317 \\
\hline Connect to blood lines aseptically & 11 & 22.9 & 24 & 50.0 & 13 & 27.1 & 10 & 20.8 & 24 & 50.0 & 14 & 29.2 & 0.317 \\
\hline Remove gloves & 48 & 100.0 & 0 & 0.0 & 0 & 0.0 & 48 & 100.0 & 0 & 0.0 & 0 & 0.0 & 1.000 \\
\hline $\begin{array}{l}\text { Keep vascular site bandage clean and } \\
\text { dry and not covered }\end{array}$ & 9 & 18.8 & 7 & 14.6 & 32 & 66.7 & 8 & 16.7 & 7 & 14.6 & 33 & 68.8 & 0.317 \\
\hline Perform hand hygiene & 34 & 70.8 & 7 & 14.6 & 7 & 14.6 & 34 & 70.8 & 7 & 14.6 & 7 & 14.6 & 1.000 \\
\hline $\begin{array}{c}\text { Total score } \\
\text { Mean \% score }\end{array}$ & \multicolumn{6}{|c|}{$\begin{array}{c}11.50 \pm 4.80 \\
38.33 \pm 16.01\end{array}$} & \multicolumn{6}{|c|}{$\begin{array}{c}11.21 \pm 4.40 \\
37.36 \pm 14.68\end{array}$} & $0.006^{*}$ \\
\hline $\begin{array}{l}\text { Poor } \\
\text { Fair } \\
\text { Good }\end{array}$ & \multicolumn{6}{|c|}{$\begin{array}{l}44(91.7 \%) \\
4(8.3 \%) \\
0(0.0 \%)\end{array}$} & \multicolumn{6}{|c|}{$\begin{array}{l}44(91.7 \%) \\
4(8.3 \%) \\
0(0.0 \%)\end{array}$} & 1.000 \\
\hline
\end{tabular}

$\mathrm{p}$ value for Wilcoxon signed ranks test

*: Statistically significant at $\mathrm{p} \leq 0.05$

Score system: $\quad$ poor $<50 \%$ fair $50 \%-<75 \% \quad$ good $\geq 75 \%$

Table (3) it is observed that only few nurses performed hand hygiene correctly at both shifts .Nearly more than half of the sample $(54.2 \%)$ were performing hand hygiene incorrectly in the morning shifts, compared with nearly three quarters $(72.9 \%)$ who didn't perform hand hygiene at all during the afternoon shift. Highly statistical significant differences in nurses' practice in both shifts where $(p=<0.001)$.

As regards placing the access limb on a sterile drape, the table shows that $12.5 \%$ of the studied nurses placed the access limb on a sterile drape correctly in both shifts. These results represented a highly statistical significant differences in nurses' practice in both shifts where $(\mathrm{p}=0.005)$. Also, the table shows that all nurses didn't wear proper PPE during both shifts. In addition, there were no significant difference in other nurses' practices regarding cannulation of AV fistula between both morning and afternoon shifts. The overall practice of the majority of nurses $(91.7 \%)$ related to AV fistula cannulation at both shifts was poor.

Table (4): Distribution of the studied nurses' practices of infection prevention and control during decannulation of AV fistula/graft through morning and afternoon shifts.

\begin{tabular}{|c|c|c|c|c|c|c|c|c|c|c|c|c|c|}
\hline \multirow{3}{*}{$\begin{array}{l}\text { Nurses' practices IPC during } \\
\text { decannulation of AV fistula/graft }\end{array}$} & \multicolumn{6}{|c|}{ Morning shift } & \multicolumn{6}{|c|}{ Afternoon } & \multirow{3}{*}{$\mathbf{p}$} \\
\hline & \multicolumn{2}{|c|}{ Not done } & \multicolumn{2}{|c|}{$\begin{array}{c}\text { Done } \\
\text { incorrectly }\end{array}$} & \multicolumn{2}{|c|}{$\begin{array}{c}\text { Done } \\
\text { correctly }\end{array}$} & \multicolumn{2}{|c|}{ Not done } & \multicolumn{2}{|c|}{$\begin{array}{c}\text { Done } \\
\text { incorrectly }\end{array}$} & \multicolumn{2}{|c|}{$\begin{array}{l}\text { Done } \\
\text { correctly }\end{array}$} & \\
\hline & No. & $\%$ & No. & $\%$ & No. & $\%$ & No. & $\%$ & No. & $\%$ & No. & $\%$ & \\
\hline Carry out hand hygiene & 15 & 31.3 & 31 & 64.6 & 2 & 4.2 & 36 & 75.0 & 10 & 20.8 & 2 & 4.2 & $<0.001^{*}$ \\
\hline Wear proper PPE & 48 & 100.0 & 0 & 0.0 & 0 & 0.0 & 48 & 100.0 & 0 & 0.0 & 0 & 0.0 & 1.000 \\
\hline Put on new sterile gloves & 44 & 91.7 & 0 & 0.0 & 4 & 8.3 & 43 & 89.6 & 0 & 0.0 & 5 & 10.4 & 0.317 \\
\hline Disconnect from blood lines aseptically & 5 & 10.4 & 32 & 66.7 & 11 & 22.9 & 5 & 10.4 & 32 & 66.7 & 11 & 22.9 & 1.000 \\
\hline $\begin{array}{l}\text { Remove needles aseptically and activate } \\
\text { needle retraction device }\end{array}$ & 5 & 10.4 & 27 & 56.3 & 16 & 33.3 & 5 & 10.4 & 27 & 56.3 & 16 & 33.3 & 1.000 \\
\hline $\begin{array}{l}\text { Clean gloves worn (patient and/or staff ) } \\
\text { to compress site for } 15 \text { minutes }\end{array}$ & 40 & 83.3 & 1 & 2.1 & 7 & 14.6 & 39 & 81.3 & 1 & 2.1 & 8 & 16.7 & 0.317 \\
\hline $\begin{array}{l}\text { Disinfect and apply sterile } \\
\text { gauze/bandage to site }\end{array}$ & 3 & 6.3 & 28 & 58.3 & 17 & 35.4 & 3 & 6.3 & 28 & 58.3 & 17 & 35.4 & 1.000 \\
\hline Remove gloves (staff and/or patient) & 40 & 83.3 & 4 & 8.3 & 4 & 8.3 & 40 & 83.3 & 3 & 6.3 & 5 & 10.4 & 0.317 \\
\hline Don't recap needles & 31 & 64.6 & 3 & 6.3 & 14 & 29.2 & 31 & 64.6 & 2 & 4.2 & 15 & 31.3 & 0.317 \\
\hline $\begin{array}{l}\text { Discard needles in sharp container and } \\
\text { other equipment in special places }\end{array}$ & 1 & 2.1 & 0 & 0.0 & 47 & 97.9 & 1 & 2.1 & 0 & 0.0 & 47 & 97.9 & 1.000 \\
\hline $\begin{array}{l}\text { Carry out hand hygiene (staff and/or } \\
\text { patient) }\end{array}$ & 35 & 72.9 & 5 & 10.4 & 8 & 16.7 & 34 & 70.8 & 5 & 10.4 & 9 & 18.8 & 0.317 \\
\hline $\begin{array}{c}\text { Total score of } \\
\text { Mean\% score of }\end{array}$ & & & $\begin{array}{r}8.15 \\
37.03 \\
\end{array}$ & $\begin{array}{l}=3.37 \\
=15.32 \\
\end{array}$ & & & & & $\begin{array}{r}7.88 \\
35.80 \\
\end{array}$ & $\begin{array}{l}3.91 \\
17.78 \\
\end{array}$ & & & $<0.001$ \\
\hline $\begin{array}{l}\text { Poor } \\
\text { Fair } \\
\text { Good }\end{array}$ & & & $\begin{array}{r}1( \\
5(1 \\
2(\end{array}$ & $\begin{array}{l}5.4 \%) \\
.4 \%) \\
2 \%)\end{array}$ & & & & & $\begin{array}{c}40(8 \\
5(10 \\
3(6\end{array}$ & $\begin{array}{l}3 \%) \\
1 \%) \\
\%)\end{array}$ & & & 0.317 \\
\hline
\end{tabular}

$\mathrm{p}$ value for Wilcoxon signed ranks test

*: Statistically significant at $\mathrm{p} \leq 0.05$ 
Vascular Access Care at Hemodialysis Unit; nurses' compliance to Infection prevention and ..

Score system: poor $<50 \%$ fair $50 \%-<75 \%$ good $\geq 75 \%$

Table (4) Nearly two third of the studied nurses (64.6\%) perform hand hygiene incorrectly in the morning shifts, compared to three quarters of them (75\%) who were not performing hand hygiene at all during afternoon shifts. Highly statistical significant differences in nurses' practice were detected between morning and afternoon shifts, where $(\mathrm{p}=<0.001)$.

Also, the table shows that all nurses were not wear proper PPE during both shifts, while nearly all of them $(97.9 \%)$ discarded needles in sharp container and the other equipment in special places. In addition; there were no statistical significant differences in the rest of nurses' practices regarding decannulation of AV fistula/graft between morning and afternoon shifts. Nurses mean percent scores of practice regarding AV fistula/graft decannulation in both shifts were poor representing $(85.4 \%, 83.3 \%)$ respectively.

Table (5): Relationship between HD nurses' practices of vascular access IPC and incidence of Blood borne diseases.

\begin{tabular}{|c|c|c|c|c|c|c|c|c|}
\hline \multirow{4}{*}{$\begin{array}{l}\text { Nurses' practice of vascular access } \\
\text { IPCP }\end{array}$} & \multicolumn{8}{|c|}{ Blood borne disease } \\
\hline & \multicolumn{4}{|c|}{ Morning shift } & \multicolumn{4}{|c|}{ afternoon shift } \\
\hline & \multicolumn{2}{|c|}{$\begin{array}{c}\text {-ve HBV } \\
(\mathbf{n}=\mathbf{3 7})\end{array}$} & \multicolumn{2}{|c|}{$\begin{array}{c}+ \text { +veHBV } \\
(n=11)\end{array}$} & \multicolumn{2}{|c|}{$\begin{array}{c}\text {-veHBV } \\
(\mathbf{n}=35)\end{array}$} & \multicolumn{2}{|c|}{$\begin{array}{c}+ \text { veHBV } \\
(n=13)\end{array}$} \\
\hline & No. & $\%$ & No. & $\%$ & No. & $\%$ & No. & $\%$ \\
\hline \multicolumn{9}{|l|}{ AV fistula cannulation } \\
\hline Poor & 35 & 94.6 & 9 & 81.8 & 33 & 94.3 & 11 & 84.6 \\
\hline Fair & 2 & 5.4 & 2 & 18.2 & 2 & 5.7 & 2 & 15.4 \\
\hline Good & 0 & 0.0 & 0 & 0.0 & 0 & 0.0 & 0 & 0.0 \\
\hline$\chi^{2}\left({ }^{\mathrm{FE}} \mathbf{p}\right)$ & \multicolumn{4}{|c|}{$1.812(0.221)$} & \multicolumn{4}{|c|}{$1.160(0.294)$} \\
\hline \multicolumn{9}{|l|}{ AV fistula decannulation } \\
\hline Poor & 32 & 86.5 & 9 & 81.8 & 28 & 80.0 & 12 & 92.3 \\
\hline Fair & 4 & 10.8 & 1 & 9.1 & 4 & 11.4 & 1 & 7.7 \\
\hline Good & 1 & 2.7 & 1 & 9.1 & 3 & 8.6 & 0 & 0.0 \\
\hline$\chi^{2}\left({ }^{\mathbf{M C}} \mathbf{p}\right)$ & \multicolumn{4}{|c|}{$1.374(0.749)$} & \multicolumn{4}{|c|}{$0.908(0.820)$} \\
\hline \multicolumn{9}{|l|}{ Overall practice } \\
\hline Poor & 32 & 86.5 & 9 & 81.8 & 29 & 82.9 & 11 & 84.6 \\
\hline Fair & 5 & 13.5 & 2 & 18.2 & 6 & 17.1 & 2 & 15.4 \\
\hline Good & 0 & 0.0 & 0 & 0.0 & 0 & 0.0 & 0 & 0.0 \\
\hline$\chi^{2}\left({ }^{\mathrm{FE}} \mathbf{p}\right)$ & \multicolumn{4}{|c|}{$0.148(0.653)$} & \multicolumn{4}{|c|}{$0.021(1.000)$} \\
\hline
\end{tabular}

$\chi^{2}, \mathrm{p}: \chi^{2}$ and $\mathrm{p}$ values for Chi square test

FE: Fisher Exact for Chi square test

MC: Monte Carlo for Chi square test

Table (5): No significant relationships were identified between overall nurses' practice of vascular access IPCP and incidence of blood borne diseases in both shifts, where $(\mathrm{P}=0.653,1.000)$ respectively.

Table (6): Relationship between HD nurses' overall practices mean percent score of HD vascular access infection prevention and control throughout morning and afternoon shifts with their years of experience and educational level .

\begin{tabular}{|c|c|c|c|c|c|c|}
\hline \multirow{2}{*}{$\begin{array}{c}\text { Years of experience/ } \\
\text { education level }\end{array}$} & \multicolumn{3}{|c|}{ Morning shift } & \multicolumn{3}{|c|}{ Afternoon shift } \\
\hline & $\begin{array}{c}\text { AV fistula } \\
\text { cannulation }\end{array}$ & $\begin{array}{c}\text { AV fistula } \\
\text { Decannulation }\end{array}$ & $\begin{array}{l}\text { Overall } \\
\text { practice }\end{array}$ & $\begin{array}{c}\text { AV fistula } \\
\text { cannulation }\end{array}$ & $\begin{array}{c}\text { AV fistula } \\
\text { Decannulation }\end{array}$ & $\begin{array}{l}\text { Overall } \\
\text { practice }\end{array}$ \\
\hline \multicolumn{7}{|l|}{ Years of experience } \\
\hline$<10$ & $30.4 \pm 18.2$ & $29.6 \pm 6.9$ & $30.05 \pm 10.2$ & $28.3 \pm 16.1$ & $27.3 \pm 8.1$ & $27.9 \pm 8.2$ \\
\hline $10<20$ & $34.4 \pm 16.3$ & $35.3 \pm 11.4$ & $34.8 \pm 10.9$ & $34.7 \pm 14.6$ & $35.3 \pm 15.5$ & $34.9 \pm 11.4$ \\
\hline $20<30$ & $46.9 \pm 4.4$ & $39.9 \pm 18.8$ & $43.9 \pm 7.9$ & $44.1 \pm 5.6$ & $36.7 \pm 20.7$ & $40.98 \pm 10.04$ \\
\hline $30<40$ & $63.3 \pm 4.7$ & $70.5 \pm 22.5$ & $66.4 \pm 6.8$ & $63.3 \pm 4.7$ & $70.5 \pm 22.5$ & $66.4 \pm 6.8$ \\
\hline $\mathbf{F}$ & $5.077^{*}$ & $5.142^{*}$ & $9.526^{*}$ & $5.528^{*}$ & $3.712^{*}$ & $8.158^{*}$ \\
\hline $\mathbf{P}$ & $0.004^{*}$ & $0.004^{*}$ & $<0.001^{*}$ & $0.003^{*}$ & $0.018^{*}$ & $<0.001^{*}$ \\
\hline \multicolumn{7}{|l|}{ Education level } \\
\hline Diploma & $35.7 \pm 15.6$ & $29.6 \pm 6.6$ & $33.1 \pm 9.11$ & $33.2 \pm 13.9$ & $26.9 \pm 7.5$ & $30.5 \pm 7.7$ \\
\hline Bachelor & $43.5 \pm 16.1$ & $51.99 \pm 16.9$ & $47.1 \pm 12.97$ & $45.6 \pm 12.87$ & $53.7 \pm 19.01$ & $49.04 \pm 11.67$ \\
\hline $\mathbf{T}$ & 1.621 & $5.112^{*}$ & $4.346^{*}$ & $2.979^{*}$ & $5.440^{*}$ & $5.749^{*}$ \\
\hline $\mathbf{P}$ & 0.112 & $<0.001^{*}$ & $<0.001^{*}$ & $0.005^{*}$ & $<0.001^{*}$ & $<0.001^{*}$ \\
\hline
\end{tabular}

$\mathrm{F}, \mathrm{p}: \mathrm{F}$ and $\mathrm{p}$ values for ANOVA test.

$\mathrm{t}, \mathrm{p}: \mathrm{t}$ and $\mathrm{p}$ values for Student $\mathrm{t}$-test.

*: Statistically significant at $p \leq 0.05$ 
Vascular Access Care at Hemodialysis Unit; nurses' compliance to Infection prevention and ..

Table (6) A positive statistical relationship between nurses' years of experience and their practice was detected, as nurses having $30<40$ years of experience had the highest mean percent practice score regarding AV fistula/graft cannulation and decannulation, at both morning and afternoon shifts where $(\mathrm{p}=\leq 0.05)$.

As regards the educational level, a positive statistical relationship was detected between nurses' education level and their practice. Bachelor graduates had higher practice mean percent score than diploma in AV fistula/graft cannulation and decannulation, at both morning and afternoon shifts, where $(\mathrm{p}=\leq 0.05)$.

\section{Discussion}

Vascular access infection control is the cornerstone of managing patients safely at HD units. Nurses should adhere to the infection prevention and control practices to achieve high quality competencies ${ }^{(9)}$.

The current study has highlighted the overall poor nurses' practices of infection prevention and control during cannulation as well as decannulation of the vascular access both at morning and afternoon shift in spite of their fair knowledge. This could be interpreted by our findings, as all of the studied nurses had received a training program related to infection control practices, but they were not supplied with the necessary infection control equipments and supplies as reported by many of the studied nurses.

In this respect, Abdelsatir (2013) emphasized that HD units should be supplied with necessary equipments and should organize adequate training on HD access care for their nursing staff to protect against infection ${ }^{(7)}$. Also, Fathalla (2009) stressed the importance of the provision of high quality equipments and supplies, needed to implement sound practice of infection control practices ${ }^{(16)}$.

Although, the majority of nurses were acquainted with the significance of hand washing, many nurses were carrying out hand hygiene inadequately on morning shifts and neglected it on the afternoon shifts either during cannulation or decannulation. This result could be referred to the lack of time, nurses' staff shortage and the workload in HD unit. At the evening shifts there were always lack of supervision by senior staff and lack of monitoring and guidance by infection control committee members. Ashour (2016) reported similar findings ${ }^{(17)}$. This result is in harmony with the study done by Ramandan (2016) and Moursy (2012) who reported that the highest percentage of their study nurses didn't comply with hand washing neither before nor after patients contact during IV fluid administration ${ }^{(18,19)}$.

PPE are compulsory to prevent cross infection. Wearing gloves are required whenever caring for a patient or touching patient's equipment. The present study revealed that all nurses used only clean gloves instead of sterile ones during the whole shifts. This result may be attributed to the cost of sterile gloves, lack of strict policies and supervision, as well as workload. The CDC (2010) emphasized the use of sterile gloves during vascular access care ${ }^{(9)}$

The findings also showed that all nurses were not use the protective measures, in spite of the good knowledge of the majority. Also, the majority of them had poor knowledge about wearing goggles or face shields. This could be due to nurses' underestimation of the significance of these protective measures and the unavailability of these supplies. The previous findings were consistent with, Higgins (2008) who reported that lack of infection control equipment and supplies was the main hindering factor to the implementation of infection control practices ${ }^{(10)}$. The WHO in this context recommended wearing PPE when splashing, spraying or splattering of blood or body fluid is expected, and during HD vascular access cannulation or decannulation (20)

Concerning care of vascular access site for prevention of infection, the present study indicated that the majority of the studied nurses in both shifts did not use to clean access arm for a minute with soap and water or antiseptic soap and placing access limb on a sterile drape or barrier was done inappropriately. In this regard the National Kidney Foundation (2011) emphasized that the nurse should wash the access site with soap and water then place the access limb on a sterile drape before cannulation to decrease the invasion of the microflora into the blood stream ${ }^{(21)}$. Girou et al (2004) and Dawood (2011) postulated that HD vascular access infections can be prevented by in service education and training programs for nurses, as they are largely responsible for the care of vascular access ${ }^{(22,23)}$.

Interestingly, the present finding revealed that no relationships were found between nurses' practices and the presence or absence of neither patients nor nurses HBV/HCV infections. Those probably could be due to nurses' belief that routine patients screening for serological evidence of hepatitis infection, as well as nurses' administration of prophylactic hepatitis B immune globulin are enough in protecting them and their patients. No doubt, the high workload at those HD units in particular may have contributed to high rates of infection transmission. In this regard, the WHO (2004) stressed on considering any patient as potentially infectious ${ }^{(20)}$. Also, Higgins (2008) emphasized that nurses should adhere to infection prevention and control practices to ensure safe working practice for all patients ${ }^{(10)}$. Also, it is recommended to manage every patient as a potential HBV/HCV carrier. Furthermore, Karkar et al (2014) advised that blood and body fluid practices should be consistently used for all patients whether infected with blood-borne pathogen or not ${ }^{(11)}$. 
Vascular Access Care at Hemodialysis Unit; nurses' compliance to Infection prevention and ..

In spite of the overall poor nurses' practice related to AV fistula cannulation and decannulation in both shifts, their overall practice was significantly better at the morning than at the evening. This could be related to the inadequate number of senior staff at the evening shifts as scheduled by the unit authority.

The current study revealed that there were statistical significant relationships between nurses' overall practices and their years of experiences, as well as their level of education .In other words, nurses with longer years of experience had better practice of infection prevention and control practices than the less experienced nurses. These findings are in line with Fawyzy ( 2016)who reported that nurses with longer years of experience had better practice of infection control precaution at HD units ${ }^{(24)}$. These results may be attributed to day to day activities that enhances nurses' experience and improves their practice. Nevertheless, Metwally et al (2016) indicated that nurses with 5 to less than 10 years of experience had higher mean percent practice score than those with more than 10 years of experience ${ }^{(25)}$.

In addition, nurses with bachelor degree had significantly higher mean percent practice score than those with diploma degree. This could be interpreted by the fact that bachelor degree nurses have more extensive undergraduate programs of infection control and microbiology than those with diploma of secondary nursing schools. In this context, Deghidi and Growder (2010) reported that education is a potential means for implementing prevention strategies as it alters perception, increases knowledge and in turn changes work practice $^{(26)}$.

Therefore, nurses at HD units must possess an appropriate knowledge, skills and experience to prevent and control infection for themselves and for their patients.

\section{Conclusion}

Based on the finding of the present study, we concluded that the overall nurses' knowledge about infection prevention and control practices during vascular access care was fair. The practices of many nurses in this regard during cannulation and decannulation of vascular access care at both shifts was poor.

\section{Recommendations}

Policies and infection control procedures at HD units should be reviewed and updated to ensure strict implementation and compliance to infection prevention and control practices

$\square \quad$ An in-service and continuing education should be established within hospitals to enhance nurses' compliance to infection control measures.

$\square \quad$ Upgrade the current systems of supervision and evaluation of nurse's staff performance in HD units to ensure higher levels of compliance to infection control practices.

Provision of standards and procedure manuals on infection control measure at HD units is mandatory.

Future similar comparative or replication studies of other HD units are needed.

Nurses perception of barriers affecting their compliance to IPCP as well as their self reported suggestions or improvements are required.

\section{References}

[1]. Kugler C, Maeding I , Russell C. Non-adherence in patients on chronic hemodialysis: An international comparison study JNEPHROL 2011; 24(03): 366-375.

[2]. Lotfy E, Sakla N, El-Naggar S. Demographic study with risk factors of hemodialysis patients in Damietta Governorate, Egypt. International Journal of Advanced Research (2015), Volume 3, Issue 10,91 - 100.

[3]. Ibrahim S. Quality of care assessment and adherence to the international guidelines considering dialysis, water treatment, and protection against transmission of infections in university hospital-based dialysis units in Cairo, Egypt. Hemodialysis International 2010; 14:61-67

[4]. Egypt Health Issues Survey 2015. Retrieved 9 December 2015, from http://dhsprogram.com/what-we-do/survey/survey-display480.cfm

[5]. Young E.J., Contreras G., Robert N.E. et al. Incidence and influencing factors associated with exit site infections in temporary catheters for haemodialysis and apheresis. Nephrology Nursing Journal, 2005, 32(1), 41-50.

[6]. Dinwiddie LC, Bhola C. Hemodialysis catheter care: current recommendations for nursing practice in North America. Nephrol Nurs J. 2010 Sep-Oct; 37(5):507-20.

[7]. Abdelsatir S. Evaluation of Nurses Awareness and Practice of Hemodialysis Access Care in Khartoum State, Sudan Arab Journal of Nephrology and Transplantation 2013 May;6(2):119-21

[8]. Alter J. \& Tokars I. Preventing transmission of infections among chronic hemodialysis patients. Nephrology Nursing Journal, 2001, 28(5), 537-585.

[9]. Centers for Disease Control and Prevention. (2010). Recommendations for preventing transmission of infections among chronic hemodialysis patients

[10]. Higgins M. Nurses' knowledge and Practice of Vascular Access Infection Control in Haemodialysis Patients in The Republic of Ireland Journal of Renal Care 2008: 47-53.

[11]. Karkar A, Bouhaha B, Dammang M. Infection Control in Hemodialysis Units: A Quick Access to Essential Elements Saudi J Kidney Dis Transpl 2014;25(3):496-519 
Vascular Access Care at Hemodialysis Unit; nurses' compliance to Infection prevention and ..

[12]. Yanai, M. Uehara, Y. and Takahashi, S.Surveillance of infection control procedures in dialysis units in Japan: a preliminary study. 2006

[13]. Storr, J. Topley, K. and Privett, S. the ward nurse's role in infection control. Nursing Standard Journal.2005, 19 (41): 56-64.

[14]. Abd-El Hady S;. Khalifa M, Zein El Dein N3, Hemdan H. Effect of an Infection Control Competency Based Protocol on the Occurrence of Blood Borne Infection in Pediatric Hemodialysis Unit at Minofiya University Hospital Asian J. Med. Pharm. Res.1(1): 26-38, 2011

[15]. Allegranzi B, and Pittet D. Healthcare-Associated Infection in Developing Countries: Simple Solutions to Meet Complex Challenges infection control and hospital epidemiology December 2007, vol. 28, no. 12

[16]. Fathalla, A. Study to Assess Nursing Interventions for the Maintenance and Prevention of Vascular Access Complications in Some Pediatric Hemodialysis Units. Unpublished Master's thesis. Minofiya University, 2009.

[17]. Ashour M. Safe nursing practices applied for patients post hip joint replacement. Unpublished Master Thesis. Faculty of Nursing , Alexandria University, Egypt 2016

[18]. Ramadan S. Nurses compliance with standard practices in intensive care units. Unpublished Master Thesis. Faculty of nursing, , Alexandria University, Egypt 2016

[19]. Moursy A. Nurses performance of infection control practices during venipuncture procedure, Alexandria Scientific Nursing Journal,2012.

[20]. World Health Organization. Practical guidelines for infection control in health care facilities.2004. Retrieved from http://whqlibdoc.who.int/emc

[21]. National Kidney Federation (England). Your Child \& Chronic Kidney Disease — an introductory manual for families — What is Kidney Disease. No. 165. May 2011.

[22]. Girou E, Buu-Hoi, ,Stephan F and Novara A.Catheter colonization in long term dialysis patients: dialysis Care Med 2004:30:22533.

[23]. Dawood, R. Effect of Implementing a Protocol of Post Operative Arteriovenous Fistula Care on the Clinical Outcome of Patients with end Stage Renal disease .Unpublished Master's Thesis. Faculty of Nursing. Alexandria University, 2011.

[24]. Fawzy N. Infection control practices of nosocomial infection at hemodialysis units, Unpuplished Master thesis, Faculty of Nursing. Alexandria University,2016.

[25]. Metwally H, Abou Donia S, Abdel Aziz T. Safety nursing measures for patients undergoing upper gastrointestinal endoscopy. Alexandria Scientific Nursing Journal,2016:18 (1):17-41.

[26]. Deghidi S and Growder D. Knowledge, awareness and compliance with universal preacuations among health care workers at the university hospital of the West Indies,Jamaica . theijoem 2010:1(4):171-8. 